(range 0-14) and twice in their last twenty-four hours (range 0-5). Hyperglycaemic episodes were recorded on two occasions and a hypoglycaemic episode was recorded once. Of those patients on oral agents (five) these were stopped between 48-120 hours before death. Of patients on insulin (four) all patients had injections in their last 24 hours of life. Conclusion Patients with diabetes make up a significant proportion of palliative populations. We currently are not discussing with patients about how best to manage their diabetes, resulting in numerous blood sugars tests, high and low blood sugars and a significant medication burden.

\section{0-17 SEARCHING FOR THE HOLY GRAIL? EXERCISE AND NUTRITIONAL REHABILITATION (ENeRgy) IN PATIENTS WITH CANCER}

${ }^{1,2}$ Charlie Hall, ${ }^{1,2}$ Barry Laird. 'St Columba's Hospice, Edinburgh, UK; ${ }^{2}$ Institute of Genetics and Molecular Medicine, The University of Edinburgh, Edinburgh, UK

\subsection{6/bmjspcare-2018-hospiceabs.17}

Background Rehabilitation is advocated as an essential component of palliative care (Tiberini \& Richardson, 2015) and is increasingly being adopted throughout various care settings. However, data on efficacy and key components of rehabilitation are lacking (Salakari, Surakka, Nurminen et al., 2015). There is a persuasive argument that any rehabilitation programme should combine exercise and nutrition, which together may improve physical function and quality of life, but this needs to be assessed (Payne, Larkin, McIlfatrick et al., 2013; Chasen, Bhargava, MacDonald, 2014).The ENeRgy trial will compare an Exercise and Nutrition based Rehabilitation programme with standard care, in patients with advanced cancer. The trial is funded by Marie Curie and the Chief Scientist Office.

Aims The primary aim of the ENeRgy trial is to assess the feasibility of an Exercise and Nutrition based Rehabilitation programme. Secondary aims will assess patient and partnercarer quality of life measures, functional and nutritional status, contamination of the control group and health economic impact.

Methods A single centre, randomised (1:1), unblinded feasibility trial is underway. Patients are randomised to receive an exercise and nutrition based rehabilitation programme (intervention) or standard care (control). Eligible patients meet the following criteria: $>18$ years; Karnofsky Performance Status $>60$; have incurable cancer; not currently undergoing anticancer treatment (bisphosphonates and hormone therapies are permitted).

Results The trial is recruiting until February 2019. Preliminary results are encouraging with 16\% recruitment and 20\% attrition rates. Patients are tolerating the individualised rehabilitation programme and positive feedback is emerging in terms of patient centred outcomes.

Conclusions The ENeRgy trial is a key step in defining, developing and assessing the feasibility of an outpatient, hospice based rehabilitation programme in this patient cohort. The results of this feasibility trial may pave the way for a wider, multi-centre trial to generate high quality evidence for rehabilitation in advanced cancer patients. This research has the potential to further guide the evolving arena of Rehabilitative Palliative Medicine.

\section{0-18 VIRTUAL REALITY DISTRACTION THERAPY IN PALLIATIVE CARE: A FEASIBILITY STUDY}

${ }^{1}$ Mark Mills, ${ }^{2}$ Simon Roughneen, ${ }^{3}$ Stephen Mason, ${ }^{1}$ Laura Chapman, ${ }^{2}$ Andrew Khodabukus,

${ }^{2,3}$ Amara Nwosu. ${ }^{1}$ Marie Curie Hospice Liverpool, Liverpool, UK; ${ }^{2}$ Academic Palliative and End of Life Care Centre, Royal Liverpool and Broadgreen University Hospitals NHS Trust, Liverpool, UK; ${ }^{3}$ Palliative Care Institute Liverpool, University of Liverpool, Liverpool, UK

\subsection{6/bmjspcare-2018-hospiceabs. 18}

Background The rapid development of technology creates opportunities to improve the delivery of healthcare. An example of a technological innovation with the potential to improve palliative care is the use of virtual reality (Bower, 2014). Previous studies have demonstrated that virtual reality (VR) is beneficial in certain situations (such as distraction therapy for pain management) and there some examples of use in hospices (Bower, 2014; Chirico, Lucidi, De Laurentiis, et al., 2016; Mahrer \& Gold, 2009; Sharar, Miller, Teeley, et al., 2008). However, to date there is little data in the literature concerning the potential benefits of VR therapy in palliative care.

Aim This project will determine the feasibility of using VR distraction therapy in specialist palliative care hospital and hospice inpatient settings.

Method This project will be conducted according to the Plan, Do, Study and Act (PDSA) quality improvement cycle. Samsung Gear VR headsets will be used to deliver the VR experience. Participants will be recruited from the Marie Curie Hospice Liverpool and the Academic Palliative Care Unit of the Royal Liverpool University Hospital. Participants will select videos from a curated content library and will be interviewed following completion of the VR session. Information about the patient experience, length of VR sessions, content choice and adverse effects will be recorded. This project is part of the Royal Liverpool and Broadgreen University Hospitals NHS Trust Global Digital Exemplar (GDE) programme and will be conducted over two months.

Expected outcomes The outcomes from the VR evaluation will help to develop future research, to study how VR can improve patient experience and support clinical care. Specifically, future work can examine whether VR-based distraction therapy can improve the symptom management for patients undergoing procedures. The outcomes of this project will be used to develop policy to support the wider adoption of VR in other hospital departments and hospices.

\section{Parallel session 6: Care settings and service design}

\section{0-19 COMMUNITY VOLUNTEERING IN ADULT HOSPICES: ANALYSIS OF THE EXTENT, BENEFITS AND BARRIERS}

${ }^{1}$ Ros Scott, ${ }^{2}$ Jean Hindmarch. ${ }^{1}$ University of Dundee, Dundee, UK; ${ }^{2}$ Hospice UK, London, UK 10.1136/bmjspcare-2018-hospiceabs.19

Background Hospice volunteers have become increasingly involved in supporting patients, families and carers in the community. There is little known about the extent, structure and benefits of such services, or of barriers to development. Hospice UK undertook a mapping survey as part of a larger project. 
Aims The aims were to:

- map the extent of hospice community volunteering services

- gather information about services already in place

- identify barriers to developing services

- inform the development of resources for hospices interested in establishing community volunteering services.

Methods A 40 item online questionnaire was developed, reviewed by the project reference group, piloted and revised. Comprising multiple choice and open-ended questions, it was circulated to the full membership of Hospice UK; 225 hospices including 14 combined adult and children's services and 25 children's hospices. Quantitative analysis was undertaken using both the online survey data output and Excel. Qualitative data were analysed using a framework approach. Children's hospice data were analysed and reported separately.

Findings from adult hospices

- 115 adult hospices responded

- $70 \%$ had community volunteering services (CVS)

- $61 \%$ of hospices without CVS were keen to develop these

- the majority of hospice CVS were staff-led.

Benefits included:

- improved wellbeing for patients, families and volunteers

- respite for families/carers

- improved access to a range of services for patients and families

- improved quality of care

- staff having greater understanding of patients' and family needs

- staff skills and time more effectively used

- increased service provision.

Barriers to development included:

- lack of resources (including funding, staff, volunteers and time)

- concern over lone-working, safeguarding and insurance.

Respondents indicated that they would find materials to guide development helpful.

Conclusions Hospice community volunteering services appear to be widespread and growing. There are clear benefits for patients, families, volunteers, organisations and staff.

\section{0-20 A REDESIGN OF DAY THERAPY SERVICES; FROM SOCIAL GROUPS TO 'SPECIALIST CARE' AND BACK AGAIN}

Emily Stowe, Sarah Thompson, Carolanne Brannan. St Clare Hospice, Hastingwood, UK

10.1136/bmispcare-2018-hospiceabs.20

Background Eight years ago Day Therapy (DT) services switched from being a mainly social/medical model, towards a therapeutic model, alongside medical care. There was a focus on providing 'specialist' palliative care and moving away from social models of care. Over time, with a changing patient population, it became apparent that this model was not meeting their needs and was providing a costly service for those with only social needs. Services were re-evaluated and a new model was developed to address the needs of our current and future patients.
Aims To move from a 'specialist' DT model towards a mixed model, including a range of outpatient opportunities and more 'light touch' services. To evaluate services including attendance figures, OACC (Outcome Assessment and Complexity Collaborative) and other outcome measures and user feedback.

Methods Some existing services maintained, alongside new services:

- Therapeutic group work for those with complex physical, emotional, social and spiritual needs, including nursing assessment and involvement of MDT as appropriate

- Rehabilitative and psychoeducational groups encouraging self management

- Increased outpatient services

- Social drop-in groups, including café style and creative social groups, delivered by a therapy assistant and volunteer.

Results Due to routine overbooking, between 96\%-120\% of available sessions were booked with attendance ranging from $46 \%$ to $101 \%$. Drop-in sessions were particularly successful, with 92\%-101\% attendance. Around 70\% of people accessing services have a non-cancer diagnosis. User feedback has been positive for sessions, including quotes such as 'supportive' and 'something to get out of bed for'. OACC data have demonstrated that people have complex, multidimensional needs. De Jong Gierveld Loneliness Scale outcomes have improved in social groups and have highlighted individuals' social needs.

Conclusion People accessing Day Therapy services have a complex mixture of physical, psychological and social needs and services need to be designed to meet these in appropriate and cost effective ways.

\section{0-21 HOW DO HOSPICES FACILITATE SOCIAL SUPPORT?}

Natasha Bradley, Mari Lloyd-Williams, Chris Dowrick. University of Liverpool, Liverpool, UK

10.1136/bmjspcare-2018-hospiceabs.21

Background Social isolation is increasing, with negative consequences for both physical and mental health. People with lifelimiting illness and their carers are at risk of inadequate social support and loneliness. Hospices support social wellbeing with services including traditional day care, support groups, befriending, multi-component interventions, and community activities. Existing research suggests that access to social support is highly valued. However, models of support used in practice are poorly documented and outcomes difficult to ascertain. The significance of social support in palliative care is under-researched.

Aim To establish an overview of hospice service models that facilitate social support for adults living in the community with life-limiting illness.

Method An online survey was developed, piloted, and disseminated to adult hospices in the UK and ROI. Questions include hospice characteristics, provision of services facilitating social support, access issues, use of patient outcomes, and availability of cost data.

Results 107 hospices responded to the survey (c. 50\% of eligible hospices). A diverse range of service models were identified. Results include descriptive statistics of the sample, categories of services identified, and salient access issues. Implications of findings for research and economic evaluation are discussed. 